\title{
Liquid-like behavior in colloidal crystals ${ }^{1}$
}

\author{
Daan Frenkel *, Peter Bladon, Peter Bolhuis, Maarten Hagen \\ FOM Institute for Atomic and Molecular Physics, Kruishan 407, 1098 SJ Amsterdam. The Netherlands
}

\begin{abstract}
The van der Waals approach to predict liquid-vapor coexistence, becomes exact in the limit of weak, long-ranged attractive forces. However, for shorter-ranged attractions, the liquid range shrinks and eventually disappears altogether. When the width of the attractive well becomes very small (less than 7\% of the diameter of particles), an iso-structural solid--solid transition, reminiscent of the liquid-vapor transition, appears in the crystalline phase. This transition, that should be experimentally observable in certain colloidal suspensions, ends in a critical point. In quasi-two-dimensional systems (c.g. confined colloids), this critical point induces the formation of a stable hexatic phase.
\end{abstract}

Keywords: Liquid-like behavior; Colloidal crystals

The first molecular theory of liquids was presented in 1873 by van der Waals in his doctoral thesis on the 'Continuity of the Gaseous and Liquid States'. In this thesis, van der Waals gave the correct explanation for a well-known yet puzzling feature of liquids and gases, namely that there is no essential distinction between the two: above a critical temperature $T_{\mathrm{c}}$, a vapor can be compressed continuously all the way to the freezing point. Yet below $T_{\mathrm{c}}$, a first-order phase transition separates the dilute fluid (vapor) from the dense fluid (liquid) [1].

The thermodynamic perturbation theory that lies at the root of the van der Waals equation, becomes exact in the limit of weak, long-ranged intermolecular interactions [2]. From the work of Longuet-Higgins and Widom [3], we know that the van der Waals model (molecules are described as hard spheres with an infinitely weak, infinitely long-ranged attraction) is even richer than originally expected: it

\footnotetext{
* Corresponding author.

1 This paper is based on material that has been published elsewhere.
}

exhibits not only the liquid-vapor transition but also crystallization.

A liquid-vapor transition is possible between the critical point and the triple point. Fig. 1 shows the full phase diagram of the van der Waals model. Yet, although the van der Waals model clearly has a liquid-vapor transition, there is no fundamental reason why this transition should occur in every atomic or molecular substance, nor is there any rule that forbids the existence of more than one fluid-fluid transition (for a discussion of the 'unexpectedness' of the liquid state, see e.g. [4]).

Whether a given compound will have a liquid phase, depends sensitively on the range of the intermolecular potential: as this range is decreased, the critical temperature approaches the triple-point temperature, and when $T_{\mathrm{c}}$ drops below the latter, only a single stable fluid phase remains. This phenomenon is well-known in mixtures of spherical colloidal particles and non-adsorbing polymer, where the range of the attractive part of the effective colloid-colloid interaction can be varied by changing the size of the polymer [5-9]. Experiment, theory and simulation 


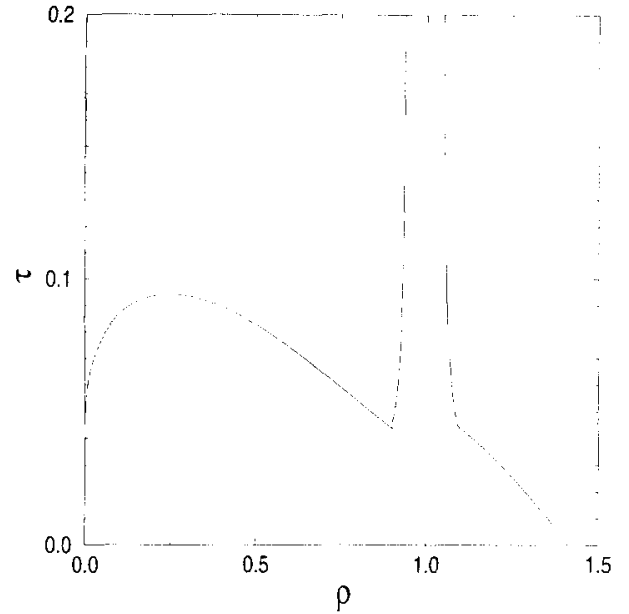

Fig. 1. Phase diagram for the van der Waals model. This phase diagram is computed by using the hard-sphere system as reference system and adding a weak, long-ranged attractive interaction. Density $\rho$ in units $\sigma^{3}$, where $\sigma$ is the diameter of the hard spheres. The "temperature' $\tau$ is defined in terms of the second virial coefficient: $B_{2} / B_{2}^{\mathrm{HS}} \equiv \mathrm{I}-1_{1}^{\prime}(4 \tau)$, where $B_{2}^{\mathrm{HS}}$ is the second virial coefficient of the hard-sphere reference system.

all suggest that when the width of the attractive well becomes less than approximately one-third of the diameter of the colloidal spheres, the colloidal 'liquid' phase disappears.

Consider, for instance, the phase behavior of an hard-core fluid with an attractive Yukawa interaction:

$u(r)= \begin{cases}\infty & (r<\sigma) . \\ -\delta \frac{\exp (\kappa \sigma(1-r / \sigma))}{r / \sigma} & (r \geqslant \sigma) .\end{cases}$

where $\sigma$ is the diameter of the hard core, $z$ is the well depth, and $k^{-1}$ is a measure for the range of the attractive part of the potential.

Computer simulations [10] show that, if the range of the attraction is larger than approximately $20 \%$ of the hard-core diameter, a liquid-vapor transition is possible. For example, Fig. 2 shows the results of a computer simulation of a Yukawa system with $\kappa \sigma=$ 3.9. In this case, liquid-vapor coexistence is clearly present. In contrast, when $k \sigma=7$ (see Fig. 3). The liquid-vapor coexistence curve has moved below the sublimation line, indicating the absence of a stable liquid range. In fact, there is even numerical evidence that in a molecular compound $\left(\mathrm{C}_{60}\right)$, the range of the

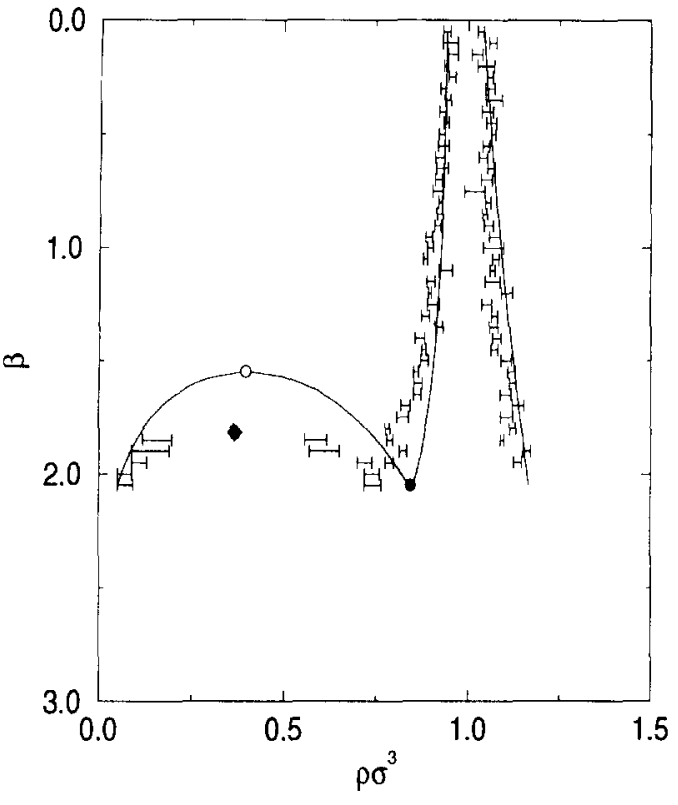

Fig. 2. Phase diagram of the hard-core attractive Yukawa system (see Eq. (1)) for $\kappa \sigma=3.9$. In this figure the open circle denotes the critical point, the filled circle the triple point and the drawn lines correspond to the results of first-order perturbation theory (see Ref. [10]). The points with error-bars are the simulation results. The diamond indicates the critical point as obtained by Gibbs-ensemble simulations.

intermolecular attraction may be sufficiently short to suppress the liquid-vapor transition [11].

Next, consider what happens in systems with a very short-ranged attraction, where the liquid-vapor transition is absent. Such systems could be realized in mixtures of uncharged colloids and short polymers. Recent computer simulations $[12,13]$ show that such systems may exhibit a solid-solid transition that is in many ways reminiscent of the liquid-vapor transition: in particular,

1. the transition takes place between two phases that have the same structure,

2. the line of (first-order) solid-solid transitions ends in a critical point, and

3. the transition depends strongly on the range of the intermolecular attraction.

As a first approximation, we use the square well potential to model short-ranged interactions in mixtures of uncharged colloids and polymers. The square-well model potential is harshly repulsive at distances less than a characteristic diameter $\sigma$ and has an attractive 


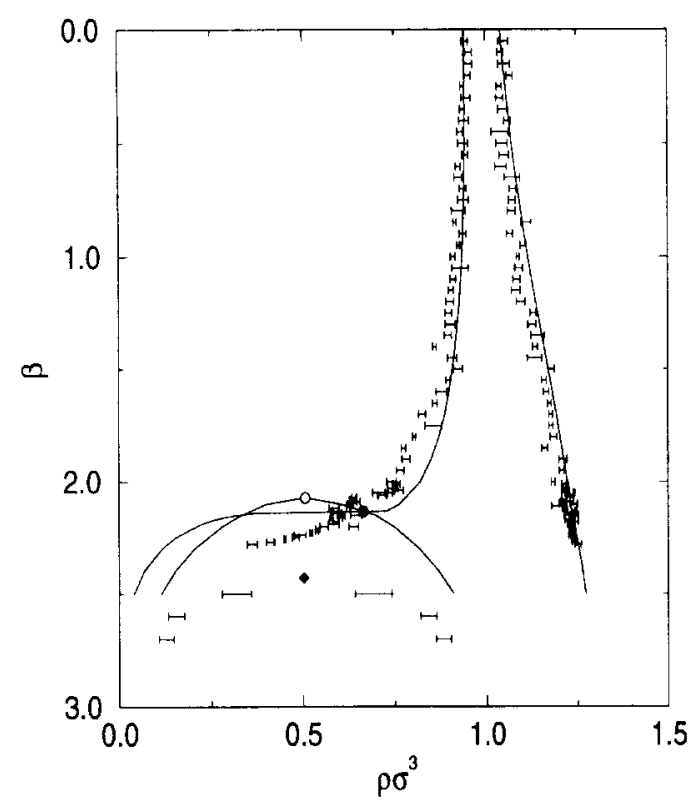

Fig. 3. Phase diagram of the hard-core attractive Yukawa system (see Eq. (1) for $\kappa \sigma=7$. In this figure the open circle denotes the critical point, the filled circle the triple point and the drawn lines correspond to the results of first-order perturbation theory (see Ref. [10]). The points with error-bars are the simulation results. The diamond indicates the meta-stable critical point as obtained by Gibbs-ensemble simulations.

interaction with a characteristic range $\delta$, outside the repulsive core. The functional form of the square-well potential is

$v(r)= \begin{cases}\infty, & 0 \leqslant r<\sigma, \\ -\varepsilon, & \sigma \leqslant r<\sigma+\delta, \\ 0, & r \geqslant \sigma+\delta .\end{cases}$

where $\varepsilon$ is the depth of the attractive well.

In fact, the occurrence of the solid-solid transition in systems with short-ranged potentials is not sensitive to the precise form of the potential and is therefore likely to be experimentally observable. Additional evidence for the insensitivity of the solid-solid transition to the precise shape of the intermolecular potential comes from recent theoretical work by Tejero et al. [14] and the simulations of Ref. [13].

It is well-known that systems with a short-ranged attraction cannot exhibit a phase transition in one dimension. However, our simulations show that such a transition is possible in two dimensions. As will be discussed below, this turns out to have interesting consequences. In order to compute the phase diagram of the square-well system, we first must determine the dependence of the Helmholtz free energy of the solid on density and temperature. As the free energy of the solid cannot be measured directly in a Monte Carlo simulation, we use thermodynamic integration to relate the free energy of the square-well solid to that of a reference hard-sphere solid at the same density [15].

In order to map out the phase diagram of the square-well solid over a wide range of densities and temperatures as a function of the width of the attractive well, several thousand independent simulations were required. To keep the computational costs within bounds, we chose to simulate a relatively small system. With a small system size, finite-size effects are expected, in particular in the vicinity of a critical point. However, away from critical points finite-size effects should be so small that they will not affect the conclusions that we draw below.

In what follows, we use reduced units, such that $\delta / k_{\mathrm{B}}$ is the unit of temperature, and $\sigma$, the hard-core diameter of the particles, is the unit of length. Figs. 4 and 5 show the computed solid-solid and fluid-solid coexistence curves in the $\rho, T$ plane for the two- and three-dimensional square-well models. We first focus on the solid--solid transition. The density gap between the dense and expanded FCC solids is wide at low temperatures and shrinks to zero when the solid-solid critical point is approached. Because of the analogy with liquid-vapor coexistence, one would expect that the solid-solid critical point should be of the $2 \mathrm{D}$ - and 3D-Ising universality class.

The coexistence curves are asymmetric, especially in the limit $\delta \rightarrow 0$. In this limit, the reduced critical temperature $T_{\mathrm{c}}$ goes to a finite limiting value of approximately 1.7 in three dimensions and 0.92 in two. This may seem surprising but, in fact, this limit can be studied directly using a peculiar lattice model [13].

The solid-solid coexistence region shifts to lower densities as the well-width is increased. This effect can easily be understood by noting that a dense square-well solid can be expanded at virtually no cost in potential energy, up to the point where the nearest-neighbor separation is $\sigma+\delta$. It is only when the solid is expanded beyond this limit that the potential energy increases steeply and a transition to the expanded solid may occur. Hence, the larger $\delta$, the lower the density where the phase transition will 


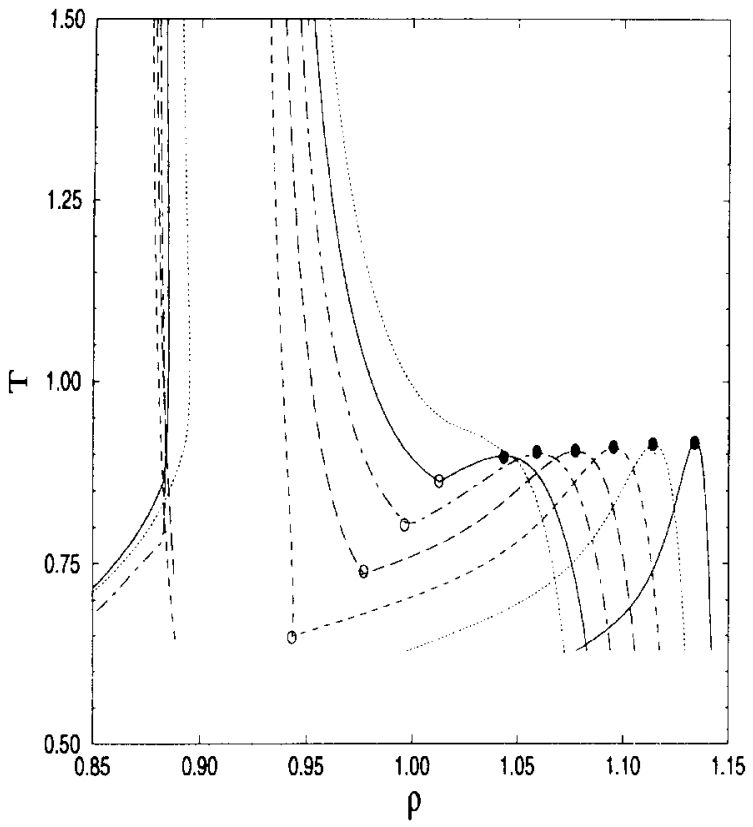

Fig. 4. Simulated $T, \rho$ phase diagrams for a two-dimensional triangular lattice of 200 square-well particles. Starting with the coexistence curve on the right, from right to left the curves correspond to the well widths $\delta / \sigma=0.01,0.02,0.03,0.04,0.05,0.06$ and 0.07 . Solid-fluid coexistence curves are shown for all systems with $\delta / \sigma \geqslant 0.03$. The critical points are indicated by filled circles, the triple points by open circles.

take place. When $\delta$ becomes larger, the vapor-solidsolid triple point shifts to higher temperatures and densities, until it reaches the critical temperature. At that point the solid-solid transition disappears because for larger values of $\delta$ it is pre-empted by the melting transition. Both in two and three dimensions, this happens when $\delta>0.06$. It should be noted that iso-structural solid--solid transitions are known to occur in dense Cs and $\mathrm{Ce}$ [17]. However, in this case the intermolecular potential is too long ranged to induce the mechanism described above and the transition is believed to be due to the softness of the intermolecular potential associated with a pressureinduced change in the electronic state of the metal ions.

The occurrence of a solid-solid critical point in two-dimensional crystals turns out to have interesting implications for the melting transition in such solids [18]. The debate about the nature of the melting transition in (quasi) two-dimensional

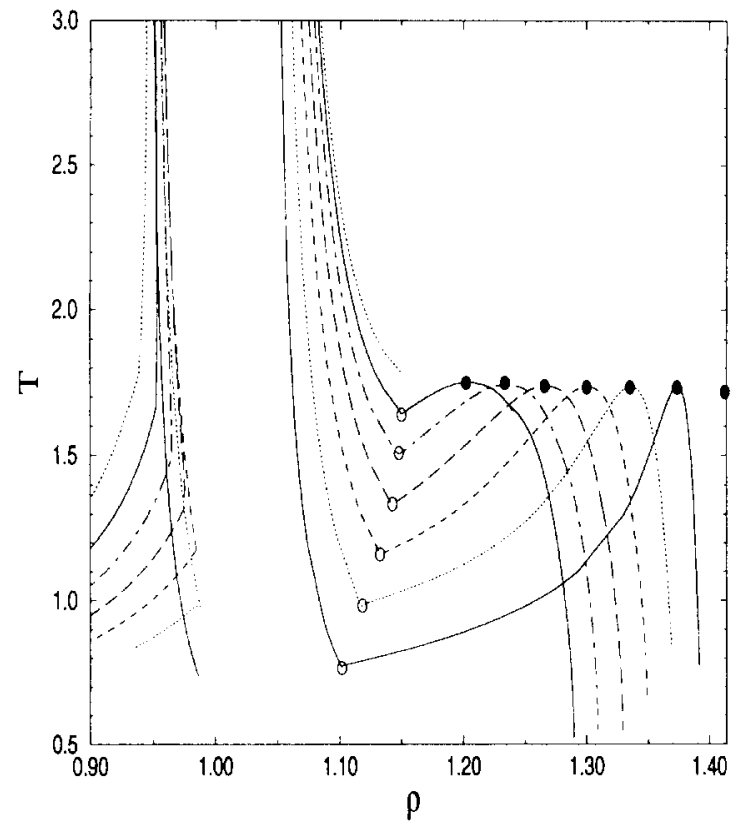

Fig. 5. Phase diagrams for a three-dimensional FCC lattice of 108 square-well particles. From right to left the curves correspond to the well widths $\delta ; \sigma=0.01,0.02,0.03,0.04,0.05$ and 0.06 . The upper dashed fluid-solid coexistence curve refers to a well width of $\delta / \sigma=0.07$ and shows that the solid-solid transition has become meta-stable at this point. Symbols as in Fig. 4. The critical point at for $\delta / \sigma=0$ was computed using the lattice model described in Ref |13].

systems dates back to the seminal work of Landau and Peierls, who showed that there is no long-ranged positional order in two-dimensional crystals (see e.g. [19]). In the early 1970s, Kosterlitz and Thouless suggested that melting in two dimensions might proceed via a continuous dislocation-unbinding transition [20]. Subsequently, Halperin and Nelson [21] argued that the phase that results after dislocation unbinding is not an isotropic liquid, as it still has quasi-long-ranged bond-orientational order. A second (disclination unbinding) transition is required to go from this bond-ordered phase, termed "hexatic" in Ref. [21], to the isotropic fluid. The continuous dislocation-unbinding transition can only occur when the dimensionless combination of elastic constants $K$ equals $16 \pi$ :

$K=\frac{4 \mu[\mu+\lambda]}{2 \mu+\lambda}=16 \pi$ 
The quantities $\lambda$ and $\mu$ are the Lame elastic constants, rendered dimensionless through multiplication by $a_{0}^{2} / k_{\mathrm{B}} T$, where $a_{0}$ is the lattice spacing.

It should be realized that the theory only predicts the point at which the solid becomes unstable to a spontaneous generation of free dislocations. The theory does not exclude the possibility that a first-order melting transition to the isotropic fluid phase intercedes at a point where the solid is still stable with respect to dislocation unbinding (i.e. for $K>16 \pi$ ). Unfortunately, in most simulation studies, the point where $K$ reaches the value $16 \pi$ is depressingly close to point where first-order melting seems to take place.

An alternative possibility is that two-dimensional solids melt by a spontaneous proliferation of grain boundaries. Fisher et al. [22] showed that the grainboundary melting should only be expected if $E_{\mathrm{c}}$, the core energy of dislocations, is not large compared to $k_{\mathrm{B}} T$. A more quantitative prediction was subsequently made by Chui [23], who argued that grain boundary proliferation is the preferred melting mechanism if $E_{\mathrm{c}}$, is less than $2.84 k_{\mathrm{B}} T$. Simulation of a defect Hamiltonian by Saito [24] confirm this picture: for $E_{\mathrm{c}}$ less than $2.84 k_{\mathrm{B}} T$, there is a first-order transition caused by a nucleation of grain boundary loops; when $E_{\mathrm{c}}$ exceeds $2.84 k_{\mathrm{B}} T$, melting takes place via a continuous transition of the Kosterlitz-Thouless type. It appears, then, that the defect core energy is the vital predictor of the melting mechanism. In the systems simulated to date, there has been no opportunity to systematically vary the defect core energy, and therefore no opportunity to explore the region of parameter space where the dislocation-unbinding theory should be valid (for a review, see e.g. $[25,26]$ ).

Extensive simulation studies of a variety of twodimensional model systems have thus far failed to provide compelling evidence for the dislocationunbinding melting scenario and some even come close to proving the opposite [25-27]. However, if we consider two-dimensional solids that exhibit a firstorder solid-solid transition ending in a critical point, we find that there is now a completely different possibility to form a hexatic phase. The reason why this should be so can be understood by considering the equation for $K$ (see $\mathrm{Eq}$. (3)). In two dimensions the bulk modulus equals $B=\lambda+\mu$ (we use the symbol $B$ for the bulk modulus to avoid confusion with the
Kosterlitz-Thouless elastic constant $K$ ). Well away from the critical point $B$ is relatively large. Close to the critical point, where $B$ vanishes,

$K=\frac{4 \mu B}{\mu+B} \sim 4 B\left(1-\frac{B}{\mu}+\cdots\right)$.

can be made arbitrarily small. Note that the shear modulus, $\mu$, is not strongly affected by a solid-solid critical point. Hence, there will be a finite region around the critical point where $K<16 \pi$ and the solid will, necessarily, become unstable with respect to dislocation unbinding.

We have studied the possibility for dislocation unbinding in the same two-dimensional square-well model that was discussed above in the context of the solid-solid transition. These simulations showed that solid-solid coexistence is possible if the width $\delta$ of the square well is less than $\sim 7 \%$ of the particle diameter. For longer-ranged attraction the triple-point temperature becomes greater than the critical-point temperature, and the low-density solid disappears.

In order to map out the regions in the phase diagram that are unstable to dislocation unbinding, we performed extensive MD simulations of the twodimensional square-well system in the vicinity of the solid-solid critical point. Having measured the elastic constants $B$ and $\mu$ as a function of density and temperature it is a simple matter to obtain $K$ and delineate the regions of the phase diagram where dislocation unbinding should occur. We find that the defect density in our simulations is always effectively zero. This greatly facilitates the numerical calculations as the simulations can be relatively short, since there is no need to equilibrate defect structures. Even for a system of 6400 particles with $\delta=0.06$, simulations show that, at the critical point, the density of defects is negligible. The low concentration of defects is, in fact, the prime feature that makes the present model a suitable candidate for exhibiting a true dislocation--unbinding transition, as it indicates the dislocation core energy must be very large. In fact by decreasing $\delta$ and moving the solid--solid transition to higher densities the core energy, $E_{c}$, can be made arbitrarily large. As there are, in practice, no defects in the system studied by simulation, the elastic constants that we measure are the 'bare' or 'unrenormalized' elastic constants of the Kosterlitz-Thouless theory. However, these represent an upper bound to 


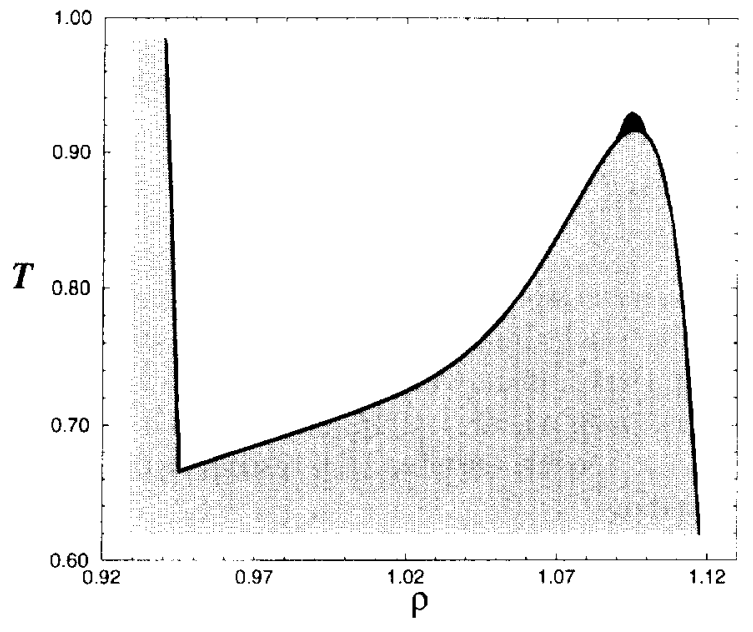

Fig. 6. The phase diagram for the $2 \mathrm{D}$ square-well system with $j=0.03$. The region of unstable solid around the solid-solid critical point - the hexatic region - is shaded black. Regions of two-phase coexistence are shaded grey. The density and temperature are expressed in units $\sigma^{-2}$ and $\iota / k_{\mathrm{B}}$, respectively. At this high density, the critical point is far from the melting line confining the hexatic region to a small area around the critical point.

the true, renormalized elastic constants of the infinite system. The presence of defects always renormalizes $K$ downwards. This is particularly obvious in that part of the phase diagram where we find $K$ to be less than $16 \pi$. In an infinite system, such values of $K$ are renormalized to zero. Hence, the range of stability of the hexatic phase will be larger than follows from the present simulations.

Fig. 6 shows the relevant part of the phase diagram for $\dot{\delta}=0.03$. The region of solid unstable to dislocation unbinding has been shaded black, and is localized in the region immediately surrounding the critical point. Fig. 7 shows the phase diagram for $\dot{\delta}=0.06$. Here the critical point is much closer to the melting line, and the triple-point temperature is much closer to the critical temperature. The lower critical density for $\delta=0.06$ causes the bulk modulus to be a much more slowly increasing function of density than is the case for $\delta=0.03$. The system is 'softer' and the region of unstable solid extends over a much larger region around the critical point. The effect of the approaching melting line can clearly be seen.

To the left of the critical point, the bulk modulus is approximately constant when compared to the rapid

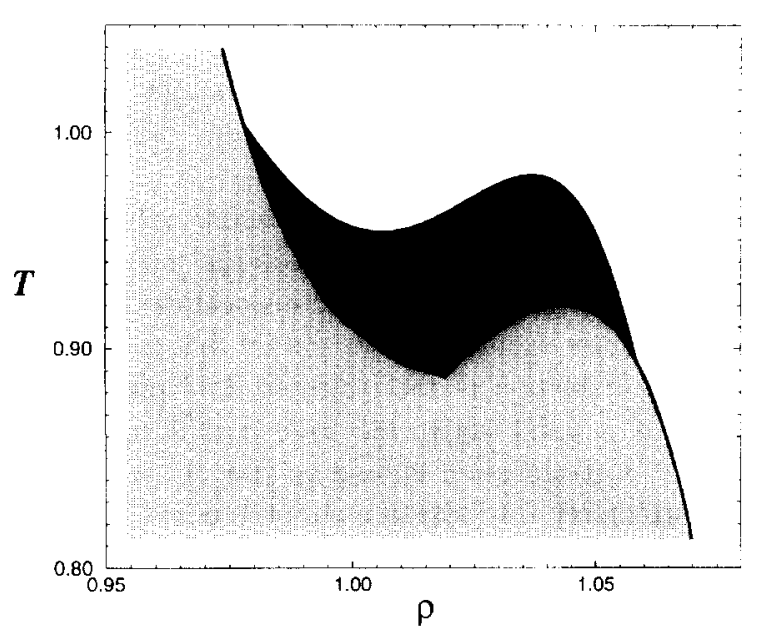

Fig. 7. The phase diagram for the $2 \mathrm{D}$ square-well system with $\delta=0.06$. The region of unstable solid around the solid-solid critical point -.. the hexatic region .- is shaded black. Regions of two-phase coexistence are shaded grey. The density and temperature are expressed in units $\sigma^{2}$ and $: k_{B}$, respectively. At this value of $\delta$ the critical temperature is close to the triple point temperature, causing the hexatic region to extend as far as the melting line.

decrease of the shear modulus with decreasing density. This lowers $K$ towards unstable values as the melting curve is approached. It would be premature to conclude that the hexatic in this region melts via a disclination unbinding mechanism, as it is quite possible that the hexatic phase undergoes a first-order transition to the isotropic fluid.

The dislocation-unbinding transition described above should be experimentally observable in quasitwo-dimensional systems, such as colloids between glass plates. There are several ways to make colloids interact through an effective potential that has a deep and narrow attractive minimum, for instance by adding small, non-adsorbing polymers. It should be noted that, even if the attractive well is too wide to induce a critical point in the solid phase, the vicinity of a critical point in the meta-stable solid should enhance the tendency towards dislocation unbinding in the stable solid. It is tempting to speculate that short-ranged attraction between charge-stabilized colloids [28] facilitates the formation of a hexatic phase in a quasi-two-dimensional system of polystyrene spheres studied by Murray and van Winkle (for a review, see [29]). 


\section{Acknowledgements}

The work of the FOM Institute is part of the research program of FOM and is made possible by financial support from the Netherlands Organization for Scientific Research (NWO). P.B. acknowledges NATO/EPSRC for support and the FOM-Institute for its hospitality.

\section{References}

[1] J.S. Rowlinson, in: Studies in Statistical Mechanics. Vol. XIV, ed. J.L. Lebowitz, (North-Holland, Amsterdam, 1988).

[2] P.C. Hemmer and J.L. Lebowitz, in: Critical Phenomena and Phase Transitions 5b, eds. C. Domb and M. Green (Academic Press, New York, 1976).

[3] H.C. Longuet-Higgins and B. Widom, Mol. Phys. 8 (1964) 549 .

[4] V.F. Weisskopf, Trans. NY Acad. Sci. Ser. II 38 (1977) 202.

[5] A.P. Gast, C.K. Hall and W.B. Russel, J. Collid. Interface. Sci. 96 (1983) 251.

[6] S. Asakura and F. Oosawa, J. Pol. Sci. 33 (1958) 183.

[7] P.N. Pusey, in: Liquids, Freezing and Glass Transition, eds. J.P. Hansen, D. Levesque and J. Zinn-Justin (North-Holland, Amsterdam, 1991) p. 763.

[8] E.J. Meijer and D. Frenkel, Phys. Rev. Lett. 67 (1991) 1110.

[9] E.J. Meijer and D. Frenkel, J. Chem. Phys. 100 (1994) 6873.

[10] M.H.J. Hagen and D. Frenkel, J. Chem. Phys. 101 (1994) 4093.

[11] M.H.J. Hagen, E.J. Meijer, G.C.A.M. Mooij, D. Frenkel and H.N.W. Lekkerkerker, Nature, 365 (1993) 425.

[12] P. Bolhuis and D. Frenkel, Phys. Rev. Lett. 72 (1994) 2211

[13] P.G. Bolhuis, M.H.J. Hagen and D. Frenkel, Phys. Rev. E 50 (1994) $4880-4890$.
[14] C.F. Tejero, A. Daanoun, H.N.W. Lekkerkerker and M. Baus, Phys. Rev. Lett. 73 (1994) 752 (see also: A. Daanoun, C.F. Tejero and M. Baus, Phys. Rev. E 50 (1994) 2913; C.N. Likos, Zs. T. Németh and H. Löwen, J. Phys. Condensed Matter 6 (1994) 10965.

[15] See e.g.: D. Frenkel. in: Molecular Dynamics Simulation of Statistical Mechanical Systems, eds. G.Ciccotti and W.G. Hoover (North-Holland, Amsterdam, 1986) p. 151.

[16] See, e.g. J.A. Barker, Lattice Theories of the Liquid State (Pergamon, Oxford, 1963).

[17] A. Jayaraman, Phys. Rev, 137 (1965) A179.

[18] P. Bladon and D. Frenkel, Phys. Rev. Lett. 74 (1995) 2519.

[19] R. Peierls, Surprises in Theoretical Physics (Princeton University Press, Princeton, 1979).

[20] J.M. Kosterlitz and D.J. Thouless, J. Phys. C 5 (1972) L124: J.M. Kosterlitz and D.J. Thouless, J. Phys. C 6 (1973) 1181 .

[21] B.I. Halperin and D.R. Nelson. Phys. Rev. Lett. 41 (1978) 121; D.R. Nelson and B.l. Halperin. Phys. Rev. B. 19 (1979) 2457; see also: A.P. Young. Phys. Rev. B. 19 (1979) 1855.

[22] D.S. Fisher. B.l. Halperin and R. Morf, Phys. Rev. B 20 (1979) 4692 .

[23] S.T. Chui, Phys. Rev. B. 28 (1982) 178.

[24] Y. Saito, Phys. Rev. B. 26 (1982) 6239

[25] For a recent review see M.A. Glaser and N.A. Clark, Adv. Chem. Phys. 83 (1993) 543 and references therein.

[26] Bond-orientational order in Condensed Matter Systems, ed K.J. Strandburg (Springer, New York, 1992).

[27] B.J. Alder and T.E. Wainwright, Phys. Rev. 127 (1962) 359; J.A. Zollweg and G.V. Chester. Phys. Rev. B. 46 (1992) 11 186: J. Lee and K.J. Strandburg, Phys. Rev. B. 46 (1992) 11 190: H.Weber and D. Marx, Europhys. Lett. 27 (1994) 593.

[28] G.M. Kepler and S. Fraden, Phys. Rev. Lett. 73 (1994) 356.

[29] See e.g. C.A. Murray in Ref. [26]. 